\title{
A Comparative Analysis of Cognitive Tutoring and Constraint-Based Modeling
}

\author{
Antonija Mitrovic ${ }^{1}$, Kenneth R. Koedinger ${ }^{2}$ and Brent Martin ${ }^{1}$ \\ ${ }^{1}$ Intelligent Computer Tutoring Group, \\ University of Canterbury, Christchurch, New Zealand \\ $\{$ tanja,brent $\} @$ cosc.canterbury.ac.nz \\ ${ }^{2}$ Human-Computer Interaction Institute, Carnegie Mellon University \\ koedinger@cmu.edu
}

\begin{abstract}
Numerous approaches to student modeling have been proposed since the inception of the field more than three decades ago. hat the field is lacking completely is comparative analyses of different student modeling approaches. Such analyses are sorely needed, as they can identify the most promising approaches and provide guidelines for future research. In this paper we compare Cognitive Tutoring to Constraint-Based Modeling (CBM). We present our experiences in implementing a database design tutor using both methodologies and highlight their strengths and weaknesses. We compare their characteristics and argue the differences are often more apparent than real. For specific domains, one approach may be favoured over the other, making them viable complementary methods for supporting learning.
\end{abstract}

\section{Introduction}

Student modeling is one of the crucial components of Intelligent Tutoring Systems (ITS). Numerous modeling approaches have been devised over the years, such as overlay modeling, enumerative bug modeling, generative and reconstructive modeling, and constraint-based modeling [4]. The ITS community acknowledges the importance of evaluation. Early ITS projects focused on the development of student modeling approaches, and rarely evaluated the methods properly. Although the percentage of papers that include evaluation results has been growing steadily, they always relate to a single student modeling approach in isolation. For the maturation of the field, it is of critical importance to perform comparative analyses of various approaches. Unfortunately, such comparative evaluations are extremely difficult; it is a major undertaking to develop any ITS, let alone two for the same domain.

In this paper we are interested in the differences between the student modeling approaches used in cognitive tutors and constraint-based tutors. We report on an initial case study in which we reimplemented a part of an existing constraint-based tutor as a cognitive tutor in order to compare and contrast the various features of these two approaches. We briefly overview cognitive tutors and constraint-based tutors. In section 4 we present the case study, followed by a comparative analysis of the two approaches. We give the conclusions in the final section. 


\section{Cognitive Tutors}

Cognitive Tutors are the most successful ITSs today [5]. Cognitive Tutors have been developed for a number of domains including algebra, geometry and LISP. They are based on the ACT-R theory of cognition [2], which claims that there are two longterm memory stores: declarative and procedural. The theory explains human learning as going through several phases. The first involves learning declarative knowledge, including factual knowledge (such as theorems in a mathematical domain), which is represented as chunks. Declarative knowledge is later turned into procedural knowledge, which is goal-oriented and therefore more efficient to use. Procedural knowledge is represented in the form of production rules. In the last phase, the production rules are further optimised when the student becomes an expert.

The fundamental assumption of ACT-R is that cognitive skills are realised by production rules. In order to support students to learn a specific task, that is, to learn a specific set of production rules that will enable students to perform the tasks correctly, cognitive tutors teach the underlying production rules.

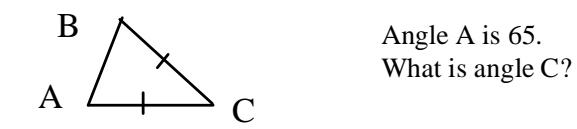

\section{Two correct production rules:}

IF goal is to find an angle in an isosceles triangle $A B C$ and $A C=A B$ and angle $A$ is known THEN set the value of angle $B$ to $A$.

IF goal is to find an angle in a triangle $A B C$ and angles $A$ and $B$ are known, THEN set the value of $C$ to $180-A-B$

Buggy production rule:

IF goal is to find an angle in an isosceles triangle $A B C$ and angle $A$ and $C$ are at the bottom of the triangle and angle $A$ is known THEN set the value of angle $C$ to $A$.

Fig. 1. Three production rules for computing the size of an angle

A generic student model is produced and used in a process called model tracing, while a student-specific model is produced by knowledge tracing. A cognitive tutor is based on a cognitive model of the domain expertise, which describes the domain knowledge needed to perform tasks like good (and perhaps poor) students. To produce this cognitive model, it is desirable to analyse how humans solve problems in a particular domain, in order to induce the underlying knowledge and represent it in the form of production rules. Cognitive tutors generate immediate feedback, i.e. they react to each step the student makes while solving a problem. An error is detected either when a student step does not match any rule, or it does match one of the buggy rules, which represent typical mistakes. Model tracing thus checks whether or not the student is performing correctly by comparing each student's step directly with one or more correct or incorrect steps that are dynamically generated by the production system. 
To illustrate, let us consider a set of production rules for finding the angles in geometry problems like the one shown at the top of Figure 1. The first two production rules can be used in sequence to first find angle B (because angles opposite equal sides are equal) and then to find angle $\mathrm{C}$ (because the sum of the angles in a triangle is 180). Once the first rule fires and finds the value for angle B, it is possible for the next rule to fire and find angle $\mathrm{C}$ (note that the angle label names are arbitrary and these rules apply to any triangle with any point labels.) The last rule in Figure 1 is an example of a buggy rule used to detect particular common mistakes. In geometry, students often over-generalize from common orientations of figures. In this case, the student thinks that the angles at the bottom of isosceles triangle are always equal.

\section{Constraint-Based Tutors}

Constraint-Based Modeling (CBM) is an approach proposed by Ohlsson [11], as a way of overcoming the intractable nature of student modeling [14]. CBM arises from Ohlsson's theory of learning from performance errors [12], which proposes that we often make mistakes when performing a task, even when we have been taught the correct way to do it. According to this theory, we make mistakes because the declarative knowledge we have learned has not been internalized in our procedural knowledge, and so the number of decisions we must make while performing the procedure is sufficiently large that we make mistakes. By practicing the task, however, and catching ourselves (or being caught by a mentor) making mistakes, we modify our procedure to incorporate the appropriate rule that we have violated. Over time we internalise all of the declarative knowledge about the task, and so the number of mistakes we make is reduced. Ohlsson describes the process of learning from errors as consisting of two phases: error recognition and error correction. After detection, an error can be corrected so that the solution used is applicable only in situations in which it is appropriate. A student needs declarative knowledge in order to detect an error. If the student does not possess such declarative knowledge, an ITS may play the role of a mentor, and inform the student of the mistake. A carefully designed sequence of feedback messages, which reflects the action of a human teacher, helps the student to overcome problems in his/her knowledge.

The starting point for CBM is that correct solutions are similar to each other in that they satisfy all the general principles of the domain. No correct solution can be arrived at by traversing a problem state that violates a fundamental principle of the domain. In CBM, we are not interested in what the student has done, but in what state they are currently in. As long as the student never reaches a state that is known to be wrong, they are free to perform whatever actions they please.

Constraints define equivalence classes of problem states. An equivalence class triggers the same instructional action, hence all states in a class are pedagogically equivalent. It is therefore possible to attach feedback messages directly to constraints. The domain model is therefore a collection of state descriptions of the form:

"If <relevance condition> is true, then <satisfaction condition> had better also be true, otherwise something has gone wrong."

In other words, if the student solution falls into the state defined by the relevance condition, it must also be in the state defined by the satisfaction condition. A violated constraint signals an error, which translates to incomplete or incorrect knowledge. 
Consider the same example of calculating angles of a triangle, used in Section 2. Figure 2 illustrates the constraints that can be used to diagnose student's solutions. The first two constraints jointly define that (only) the two base angles in an isosceles triangle have the same size. The third constraint is equivalent to the second rule in Figure 1. Constraint 2 catches the same error as the buggy rule from Figure 1.

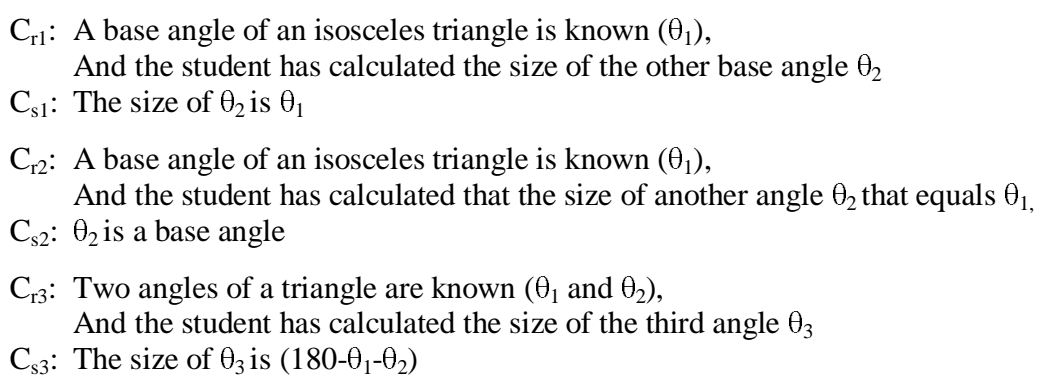

Fig. 2. Three constraints that check whether the size of an angle is correct

First, all relevance patterns are matched against the problem state. Then, the satisfaction components of constraints that matched the problem state in the first step (i.e., the relevant constraints) are tested. If a satisfaction pattern matches the state, the constraint is satisfied. Otherwise, it is violated. The short-term student model consists of all violated constraints.

We believe that CBM is also neutral with respect to the domain. Within the ICTG group, we have developed SQL-Tutor, a tutor for teaching SQL, a declarative language [10], CAPIT, a system that teaches the rules of punctuation and capitalization in English [8], KERMIT, a system for database design [15], and NORMIT [9], an ITS that teaches data normalization, which is a procedural task. We have experienced no problems expressing the knowledge in these domains in terms of constraints. CBM is applicable both to procedural and declarative domains. Currently, we are considering other domains with different characteristics, to further test the generality of CBM.

CBM, as proposed in [11], is a method for diagnosing students' solutions. The approach identifies errors, which is extremely important for students lacking declarative knowledge, because they are unable to detect errors themselves. As stated earlier, one of the goals of our research is to evaluate how well CBM supports learning. We showed how CBM can be extended to allow for long-term modeling of students' knowledge, and alternatives for generation of pedagogical actions [7].

\section{Case Study: Teaching Database Design}

In this case study we re-implemented a part of KERMIT using the cognitive tutors methodology. This allows us to compare the two approaches. We start by briefly introducing KERMIT, the context of the study, and then present our experiences.

\subsection{KERMIT: a Constraint-based Tutor for Database Design}

KERMIT (Knowledge-based Entity Relationship Modeling Intelligent Tutor), an ITS that teaches database design, was developed at the ICTG group. One of the goals of the system was to test the methodology for building ITSs that has been used at ICTG 
over the years, as database design is a domain whose characteristics are very different from those of the domains previously worked on. Database design is an open-ended task: although there is an outcome defined in abstract terms, there is no single "correct" procedure to obtain that outcome. For a detailed discussion of the system, see [15]. KERMIT is a problem-solving environment in which students practice database design using the Entity Relationship (ER) data model. KERMIT consists of an interface, a pedagogical module, which determines the timing and content of pedagogical actions, and a constraint-based modeller, which analyses student answers and generates student models. The interface displays the current problem and provides controls for stepping between problems, submitting a solution and selecting the level of feedback. It also contains the main working area, in which the student draws the ER diagram. Feedback is presented on request. KERMIT contains a set of problems and the ideal solutions to them, but has no problem solver. In order to check the correctness of the student's solution, KERMIT compares it to the correct solution using domain knowledge represented in the form of more than 90 constraints. The constraints cover both syntactic and semantic knowledge. The syntactic constraints are concerned with syntactic details in a student's solution. An example of such a constraint is "A regular entity must have at least one key attribute." Semantic constraints relate the student's solution to the system's ideal solution. For example, there are constraints that check for equivalent, but not identical, ways of modeling a database in the student's and ideal solution.

\subsection{Re-implementing KERMIT as a Model-Tracing Tutor}

To compare these two approaches, we implemented a subset of KERMIT using model tracing. We refer to this implementation as KERMIT-MT. Table 1 summarizes the differences between the two implementations. In the following discussion we will use this ER modeling problem: "Some students live in student halls. Each hall has a unique name, and each student has a unique number."

Let us start with the problem representations. KERMIT requires the problem text to be stored together with tags that identify phrases corresponding to the constructs in the ideal solution. For example, the word "student" would have a tag that identifies it as corresponding to an entity type in the ideal solution. The ideal solution and the student's solution are represented in the same way. There is a list of entities, where each entity is described in terms of its name, type and a list of attributes. Similarly, there is a list of relationships, containing the name of each relationship, its type, the names of participating entities and possibly a list of attributes.

KERMIT-MT, on the other hand, requires the problem text and additional structures. The teacher breaks the problem text into a set of clauses, where each clause is a sentence or a part of a sentence having special importance for ER modeling. For example, a clause may describe an entity, relationship or an attribute, or specify integrity rules on them. For the above problem, the text is broken into three clauses. The first clause consists of the first sentence, while the second sentence is divided into two clauses. In addition, the teacher needs to specify the working memory elements (WMEs) necessary to solve the problem, by describing all relevant words (nouns, verbs, or modifiers) appearing in the problem text. For the above problem, the teacher defines WMEs for the following nouns (noun phrases): students, halls, hall name, and student number. For each of them, the teacher further specifies 
its type (e.g. a set of objects), and possible names students would be able to use in the ER diagram. The following verbs also need WMEs to be defined: live in and has (two different instantiations). Then, the teacher defines modifiers, which are important for determining the integrity rules; in this problem there is only one (some). However, the text of the problem does not contain everything the student needs in order to solve the problem. Many of the elements come from the student's world knowledge. In KERMIT-MT, such elements were specified explicitly in order to enhance the ability to give advice. In the case of the above problem, the teacher specifies three such elements: that many students may live in one hall; that all halls have students living in them; and, finally, that a student may live in one hall only.

Table 1. A comparison of two implementations

\begin{tabular}{|l|l|l|}
\hline Feature & KERMIT-MT & KERMIT \\
\hline Problem representation & $\begin{array}{l}\text { Text + WMEs (words + world } \\
\text { knowledge) }\end{array}$ & Text + tags \\
\hline Solution representation & $\begin{array}{l}\text { Entities, attributes, relationships, } \\
\text { connections }\end{array}$ & Entity and relationship lists \\
\hline Ideal solution & Not stored & Entity and relationship lists \\
\hline Domain knowledge & $\begin{array}{l}\text { Production rules: } \\
\text { Entities: } 2 \\
\text { Attributes: } 4 \\
\text { Relationships: } 14\end{array}$ & $\begin{array}{l}\text { Matching constraints: } \\
\text { Entities: } 5 \\
\text { Done: } 5\end{array}$ \\
& $\begin{array}{l}\text { Attributes: } 9 \\
\text { Relationships: } 9\end{array}$ \\
\hline
\end{tabular}

KERMIT requires an ideal solution to be stored, while there is no such requirement in KERMIT-MT. However, it is easier to create a representation of the solution than to specify all the WMEs needed in KERMIT-MT. On the other hand, the solution representation has less information and thus less to draw on in creating meaningful advice to students.

Finally, let us discuss the domain knowledge in these two systems. KERMIT-MT covers only a part of the domain covered by KERMIT, so in Table 1 we include only that part of the domain (KERMIT currently contains over 90 constraints, but covers the complete ER domain). KERMIT-MT has 25 production rules, which cover simple and key attributes, regular entities and regular binary relationships only. These were written by Koedinger in about 20 hours and could be refined to fewer rules with more time. There are 23 constraints in KERMIT that correspond to KERMIT-MT's rules. However, these constraints are much more general, as they deal with all kinds of attributes (including composite and multivalued), relationships and entities, so they actually cover more of the domain than the rules in KERMIT-MT. Furthermore, KERMIT-MT uses buggy rules in order to generate error-specific feedback to students, while KERMIT does not.

\section{Discussion}

Table 2 summarizes the main differences between MT and CBM. The learning theories that underlie these approaches-ACT-R (CT), and "learning from performance errors" (CBM) - are both based on the distinction between declarative and procedural knowledge, and the view that learning consists of two main phases: in the first, declarative knowledge is encoded; in the second phase, this declarative knowledge is turned into more efficient procedural knowledge. The difference 
between the theories is in the amount of effort that is assumed in each phase, and also in the focus of instruction based on each theory. ACT-R assumes that the encoding of declarative knowledge is a straightforward process, where experiences are stored in an unchanged form, e.g. examples, successes and failures of attempts. Therefore, efforts are needed in the second phase, when declarative knowledge is proceduralized. In contrast, Ohlsson [11,12] claims that we make mistakes if we do not have sufficient declarative knowledge to detect errors. Consequently, cognitive tutors tend to focus on generative knowledge (production rules), while constraint-based tutors teach evaluative knowledge (constraints). However, more recent cognitive tutors have successfully addressed evaluative knowledge [6] and declarative knowledge more generally [1]. Other researchers also stress the importance of declarative knowledge. For example, Chi et al [3] see the performance in problem solving as largely determined by the completeness of the declarative knowledge, rather than the efficiency of the procedural knowledge.

MT tutors represent domain knowledge as production rules. This knowledge has high cognitive fidelity, because it is an explicit model of the reasoning that the learner must acquire. Such domain knowledge is not necessarily equivalent to an expert's knowledge, since Cognitive Tutors may require students to specify intermediate steps that human experts usually skip. High cognitive fidelity is a strong advantage of Cognitive Tutors.

CBM tutors, on the other hand, represent domain knowledge in a declarative form. Constraints cannot directly be used to solve problems; their main function is to distinguish between correct and incorrect solutions. It is interesting to note similarities between constraints and the inference rules Chi and colleagues discuss in [3]: they find that students who engage in self-explanation learn better, as a consequence of forming inference rules. Chi states that inference rules are more operational than general principles conveyed in traditional instruction, because the conditions are more specific, and inference rules are more decomposed than the general principles. The same reasoning applies to constraints. It takes a number of constraints to equal a general principle of a domain. Each constraint focuses on just one aspect of a general principle, thus allowing very specific feedback to be generated.

Model tracing tutors have been criticised for being too rigid, in that they force students to follow a fixed set of desirable approaches to solving problems [16]. For example, the Lisp tutor requires that the top-down approach be used for writing functions. One might speculate that this may be more beneficial to novices and less so for more knowledgable students, however, evaluations of the LISP tutor have tended to show fairly uniform learning improvements for students at all levels. In other words, the evidence suggests the LISP tutor does work well both for novice and more knowledgable students. On the other hand, CBM neither imposes nor supports any particular strategy, since it evaluates the current state in problem solving (as opposed to the current action which is evaluated in Cognitive Tutors). By ignoring the procedures used to solve problems, CBM allows for inconsistencies in problemsolving strategies. The downside is that CBM systems typically are not capable of strategic planning advice.

Typical CBM-based systems generate instructional actions without being able to solve problems, by comparing the ideal solution (specified by the human teacher) to the student's solution. If there are alternative solutions, they are recognized as such by 
constraints that check for the necessary elements in the solution. However, CBM does not prevent us from having a problem solver. We have developed an extension to CBM that allows problems to be solved (and student solution errors to be corrected) directly from the constraint set, and implemented it for SQL-Tutor [7]. However, it requires that the constraint set be more complete, otherwise erroneous solutions may be generated, but has the benefit of being optional. In contrast, Cognitive Tutors typically are able to solve problems and having some simulation of problem solving is important for providing planning advice. However, for domains for which it will be extremely hard, or even impossible to build problem solvers (because there is no obvious problem solving strategy), it is possible for Cognitive Tutors, like CBM, to store solutions (or approximations thereof) and write rules that work from them. In both cases, an incremental development strategy is possible whereby one starts by implementing the ITS using stored solutions and then adds problem solving capabilities as needed (ideally driven by student usage data).

Table 2. Comparative analysis of CBM and MT

\begin{tabular}{|l|l|l|}
\hline Property & Model Tracing & Constraint-Based Modeling \\
\hline Knowledge representation & Production rules (procedural) & Constraints (declarative) \\
\hline Cognitive fidelity & Tends to be higher & Tends to be lower \\
\hline What is evaluated & Action & Problem state \\
\hline Problem solving strategy & Implemented ones & Flexible to any strategy \\
\hline Solutions & $\begin{array}{l}\text { Tend to be computed, but } \\
\text { can be stored }\end{array}$ & $\begin{array}{l}\text { One correct solution stored, } \\
\text { but can be computed }\end{array}$ \\
\hline Feedback & $\begin{array}{l}\text { Tends to be immediate, but } \\
\text { can be delayed }\end{array}$ & $\begin{array}{l}\text { Tends to be delayed, but can } \\
\text { be immediate }\end{array}$ \\
\hline Problem-solving hints & Yes & $\begin{array}{l}\text { Only on missing elements, } \\
\text { but not strategy }\end{array}$ \\
\hline Problem solved & 'Done' productions & No violated constraints \\
\hline Diagnosis if no match & Solution is incorrect & Solution is correct \\
\hline Bugs represented & Yes & No \\
\hline Implementation effort & $\begin{array}{l}\text { Tends to be harder, but can } \\
\text { be made easier with loss of } \\
\text { other advantages }\end{array}$ & $\begin{array}{l}\text { Tends to be easier, but can } \\
\text { be made harder to gain other } \\
\text { advantages }\end{array}$ \\
\hline
\end{tabular}

Cognitive Tutors typically offer immediate feedback (usually only implicitly by "flagging" an error when it occurs), while constraint-based tutors typically provide feedback on demand. However, both approaches are capable of providing the other type of feedback, so this difference is somewhat superficial. Further, Cognitive Tutors can offer strategic problem-solving hints in terms of the next step to perform in a plan. These hints are typically provided on demand or after the student has made more errors on a goal than a teacher-set error threshold. They are generated by running the production set. Constraint-based tutors are, in general, not necessarily able to solve problems, but they can provide feedback on missing elements of the solution.

Another important issue is the completeness of the knowledge base. It is widely accepted that the quality of the knowledge base is the determining factor for the quality of instruction and diagnosis. In model tracing, an incomplete knowledge base may mean that there are some correct or buggy rules missing. When a student performs a step that matches neither a correct or buggy rule, that step is assumed to be incorrect. While the system cannot explain why (because there is not buggy rule), it 
is able to point the particular step in the whole solution that is in error. Cognitive Tutor developers work hard to avoid it, but it possible that a correct rule is missing in such a case that that student's step is actually correct (e.g., part of a strategy not implemented in the production rules). Thus, it is possible in Cognitive Tutors that a correct solution is rejected. In CBM, the default diagnosis in the case of no match is that the student's solution is correct. The default behaviour is "Innocent until proven guilty", versus "Guilty until proven innocent" in Cognitive Tutors. If a CBM is missing constraints, it may fail to identify faults in a student's solution and thus a student may come away thinking they know how to do something when in fact they do not. In both cases, it is important to do careful engineering and iterative student testing to prevent such situations.

Although the approach of modeling all possible solutions works well for welldefined domains such as mathematics, it may not be a realistic solution when the domain is ill defined. However, as discussed above, this purported difference between CBM and Cognitive Tutors is more apparent than real. The task of composing a collection of buggy rules is also a major undertaking. Studies have shown that bug libraries do not transfer well to new population of students; if a bug library is developed for a certain group of students, it may not cover the bugs that another group of students may make [13]. Unlike cognitive tutors, CBM does not require extensive studies of student bugs, which is an important trade-off. The down side is that CBM cannot provide feedback specific to these particular errors.

\section{Conclusions}

This paper presented a comparative analysis of two student modeling approaches: model tracing and constraint-based modeling. We discussed the characteristics and the underlying learning theories of these two approaches, and presented a case study where two tutors were developed for the same domain. We also analysed the two approaches in terms of their main attributes. Creating constraint-based modeling systems tends to require less time and effort, but the result tends to be less comprehensive in terms of specific advice-giving capabilities. Creating model-tracing tutors tends to require more time and effort, but tends to result in more specific advice-giving capabilities. This is apparent from the case study where it was arguably harder to develop a model-tracing tutor for database design than a constraint-based system. On the other hand, the model-tracing tutor has capabilities to give planning hints and advice expressed more in terms of what a student needs to think to generate a part of the answer than in terms of the desired features of that part of the answer. We emphasize that the stated differences are tendencies and are not hard and fast. It is possible to write constraint-based systems that generate solutions (ref Brent) and thus can be used to provide planning advice. Of course, doing so takes more time and effort. Conversely, it is possible to more quickly and easily build a model-tracing tutor by writing productions in a diagnostic mode that focus more on whether student steps are correct or not and less on how to generate those steps. But, again, such a change does not come without cost. The resulting cognitive tutor will not be able to provide planning advice.

We conclude that both approaches have their strengths and weaknesses. Model tracing is an excellent choice for domain where appropriate problem solving strategies are well-defined, and where comprehensive feedback on them is desirable. On the 
other hand, CBM offers a workable alternative when such strategies are not available or appropriate, or there is too little time or resources to build a model-tracing knowledge base. CBM and model-tracing are viable, complementary approaches to building real-world tutors.

\section{Acknowledgments}

KERMIT was developed by Pramuditha Suraweera. The authors thank the Erskine fund of the University of Canterbury for funding the visit of Ken Koedinger to New Zealand. The work presented here was supported by the University of Canterbury research grant U6430. This research could not have been done without the support of past and present member of HCII and ICTG.

\section{References}

1. Aleven, V., Koedinger, K.: An Effective Metacognitive Strategy: Learning by Doing and Explaining with a Computer-based Cognitive Tutor. Cognitive Science 26 (2002) 147-179

2. Anderson, J. R., Lebiere, C.: The Atomic Components of Thought. Mahwah, NJ: Erlbaum (1998)

3. Chi, M. T. H., Bassok, M., Lewis, W., Reimann, P., Glaser, R.: Self-Explanations: How Students Study and Use Examples in Learning to Solve Problems. Cognitive Science, 13 (1989) 145-182

4. Holt, P., Dubs, S., Jones, M., Greer, J.E.: The State of Student Modeling. In: Greer, J.E., McCalla, G.I. (eds.): Student Modeling: the Key to Individualized Knowledge-based Instruction. NATO ASI Series, Vol. 125. Springer (1994) 3-35

5. Koedinger, K. R., Anderson, J. R., Hadley, W. H., Mark, M. A.: Intelligent Tutoring Goes to School in the Big City. Int. J. Artificial Intelligence in Education, 8 (1997) 30-43

6. Mathan, S., Koedinger, K.: An Empirical Assessment of Comprehension Fostering Features in an Intelligent Tutoring System. In: S. Cerri, G. Gouarderes, F. Paraguacu (eds.) Proc. ITS 2002, LNCS Vol. 2363 Springer-Verlag, (2002) 330-343

7. Martin, B, Mitrovic, A.: Tailoring Feedback by Correcting Student Answers. Proc. ITS'2000, LNCS Vol. 1839, Springer-Verlag, (2000) 383-392

8. Mayo, M., Mitrovic, A.: Optimising ITS Behavior with Bayesian Networks and Decision Theory. Int. J. Artificial Intelligence in Education, 12 (2001) 124-153

9. Mitrovic, A.: NORMIT, a Web-enabled Tutor for Database Normalization. Kinshuk, R. Lewis, K. Akahori, R. Kemp, T. Okamoto, L. Henderson, C-H Lee (eds.) Proc. ICCE 2002 (2002) 1276-1280

10. Mitrovic, A., Ohlsson, S.: Evaluation of a Constraint-Based Tutor for a Database Language. Int. J. on Artificial Intelligence in Education 10 (3-4) (1999) 238-256

11. Ohlsson, S.: Constraint-based Student Modeling. In Student Modeling: the Key to Individualized Knowledge-based Instruction. Springer (1994) 167-189

12. Ohlsson, S.: Learning from Performance Errors. Psychological Review 103 (1996) 241262

13. Payne, S., Squibb, H.: Algebra Mal-rules and Cognitive Accounts of Errors. Cognitive Science, 14 (1990) 445-481

14. Self, J. A.: Bypassing the Intractable Problem of Student Modeling. In: C. Frasson and G. Gauthier (eds.), Intelligent Tutoring Systems: at the Crossroads of Artificial Intelligence and Education. Norwood: Ablex (1990) 107-123

15. Suraweera, P., Mitrovic, A.: KERMIT: a Constraint-based Tutor for Database Modeling. In: S. Cerri, G. Gouarderes, F. Paraguacu (eds.) Proc. ITS 2002, LNCS Vol. 2363 Springer-Verlag, (2002) 377-387

16. VanLehn, K. et al.: Fading and Deepening: the Next Steps for Andes and other ModelTracing Tutors. In: Proc. ITS'2000, LNCS Vol. 1839, Springer-Verlag, (2000) 474-483 